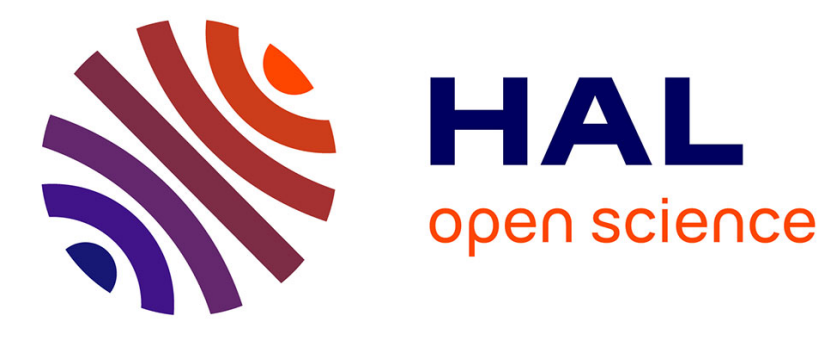

\title{
Selenium-regulated hierarchy of human selenoproteome in cancerous and immortalized cells lines
}

\author{
Zahia Touat-Hamici, Anne-Laure Bulteau, Juliusz Bianga, Hélène
}

Jean-Jacques, Joanna Szpunar, Ryszard Lobinski, Laurent Chavatte

\section{- To cite this version:}

Zahia Touat-Hamici, Anne-Laure Bulteau, Juliusz Bianga, Hélène Jean-Jacques, Joanna Szpunar, et al.. Selenium-regulated hierarchy of human selenoproteome in cancerous and immortalized cells lines. Biochimica et Biophysica Acta (BBA) - General Subjects, 2018, 1862, pp.2493 - 2505. 10.1016/j.bbagen.2018.04.012 . hal-03133602

\section{HAL Id: hal-03133602 https://hal.science/hal-03133602}

Submitted on 13 Feb 2021

HAL is a multi-disciplinary open access archive for the deposit and dissemination of scientific research documents, whether they are published or not. The documents may come from teaching and research institutions in France or abroad, or from public or private research centers.
L'archive ouverte pluridisciplinaire HAL, est destinée au dépôt et à la diffusion de documents scientifiques de niveau recherche, publiés ou non, émanant des établissements d'enseignement et de recherche français ou étrangers, des laboratoires publics ou privés. 
Selenium-regulated hierarchy of human selenoproteome in cancerous and immortilized cells lines

\section{Zahia Touat-Hamici ${ }^{1}$, Anne-Laure Bulteau ${ }^{2,3}$, Juliusz Bianga ${ }^{2}$, Hélène Jean-Jacques ${ }^{1}$, Joanna} Szpunar $^{2}$, Ryszard Lobinski ${ }^{2}$ and Laurent Chavatte ${ }^{2,4,5,6, *}$

${ }^{1}$ From the Centre de Génétique Moléculaire, CGM, CNRS, UPR3404, Gif-sur-Yvette, France.

${ }^{2}$ CNRS/UPPA, Institut des Sciences Analytiques et de Physico-chimie pour l'Environnement et les Matériaux, IPREM-UMR5254, 64053 Hélioparc, Pau, France

${ }^{3}$ Institut de Génomique Fonctionelle de Lyon, IGFL, CNRS/ENS UMR5242, 69007 Lyon, France

${ }^{4}$ Centre International de Recherche en Infectiologie, CIRI, 69007 Lyon, France

${ }^{5}$ INSERM U1111, 69007 Lyon, France

${ }^{6}$ CNRS/ENS/UCBL1 UMR5308, 69007 Lyon, France.

Running title : Selenoproteome regulation in human cell lines

* To whom correspondence should be addressed: Laurent Chavatte, Centre International de Recherche en Infectiologie, CIRI, 69007 Lyon, France, Tel.: (+33) 4727288 93; E-mail: laurent.chavatte@ens-lyon.fr

Keywords: Selenoprotein hierarchy / glutathione peroxidase / thioredoxin reductase / ICP-MS /

Background: Selenium is inserted as selenocysteine into an important group of antioxidant enzymes, the selenoproteins.

Results: We report how selenoprotein expression responds to selenium levels variation in human cell lines.

Conclusion: The selenoproteome is differentially regulated using cell line specific hierarchy.

Significance: We describe a comprehensive study at the levels of protein, mRNA and translational efficiency.

\footnotetext{
ABSTRACT

Selenium (Se) is an essential trace element that is co-translationally incorporated in selenoproteins as a rare amino-acid, selenocysteine, using an unusual UGA-recoding mechanism. The human selenoproteome, which is encoded by 25 selenoprotein genes, is highly regulated by $\mathrm{Se}$ bioavailability and tissuespecific hierarchy. Selenium deficiency is often evoked in the context of cancer, cardiac function, muscular disorders, neurodegenerative disease
}

and aging. In this report, we investigated how selenoprotein expression responds to changes in the Se concentrations in the culture medium at the protein, mRNA, amount of incorporated Se and translational efficiency levels. We analyzed four different cell lines originated from kidney (HEK293), prostate (LNCaP), skin (HaCaT) and liver (HepG2) which are of particular interest from the point of view of the Se-related health concerns. Then, to analyze the hierarchy of the selenoprotein expression in response to changes in Se concentration, we used two complementary techniques: (i) a classical semi-quantitative immunodetection method using commercial antibodies, and (ii) a novel approach based on Laser Ablation - Inductively Coupled Plasma Mass Spectrometry (LA-ICP MS) after nonradioactive ${ }^{76} \mathrm{Se}$ labeling of selenoproteins. With both methods, we found cell-line specific alterations of the selenoproteome in response to a change in Se concentration. In most of the cases, selenoprotein mRNA levels remained constant suggesting a translational control of gene expression. It follows that the nature of the 
SECIS element, present in the 3'UTR of selenoprotein mRNAs, determines the response of HEK293 cells to changes of the Se concentration in the culture medium

\section{INTRODUCTION}

Selenium is an essential trace element for human health, associated with muscular, thyroid, immune and brain function (REFS). In many regions of the globe, the selenium dietary intake is insufficient. Many epidemiological and intervention studies recognize the role of $\mathrm{Se}$ deficiency in the increased risk of cancers, including those of lung, colon and prostate. Most of the beneficial effects of Se are awarded to its incorporation as selenocysteine into a small but crucial group of proteins, the selenoproteins. The human genome encodes twenty five selenoprotein genes, several of them being ubiquitously expressed. Well characterized selenoproteins include glutathione peroxidases (Gpxs), thioredoxin reductases (TrxRs), methionine sulfoxide reductase B1 (MsrB1, or SelenoX), and selenophosphate synthetase 2 (Sephs2). They are essential components of antioxidant defense, redox signaling and redox homeostasis. However, about one third of selenoproteins remains without precise role or substrates, but are expected to function in redox reaction.

A remarkable mechanism is involved in selenoprotein biosynthesis, since selenocysteine is genetically encoded by UGA, and therefore co-translationally inserted into the polypeptide chain. Note that UGA is generally used as a stop codon for other cellullar mRNAs. A selenocysteine insertion sequence (SECIS) is present in the 3'UTR of selenoprotein mRNAs to direct faithful recoding of UGA as selenocysteine. The SECIS recruits several factors, including SECIS-binding protein-2 (SBP2), selenocysteine-specific elongation factor (EF-Sec), Sec-tRNA ${ }^{[\mathrm{Ser}] \mathrm{Sec}}$, and others reviewed elsewhere (Bulteau et al) (REFS Chavatte, budiman, nucleolin). However, despite much effort, the precise mechanism remains elusive and more intricate than anticipated with many layers of regulation.

Selenoprotein expression is mainly controlled at the level of translation, with the UGA-Sec recoding event being the rate-limiting stage. It now appears that the two central RNA components, namely the SECIS and the SectRNA $^{[\mathrm{Ser}] \mathrm{Sec}}$, govern the efficiency and fidelity of this process (REFs review). Based on luciferase reporter constructs, it was shown that the SECIS elements display a wide range of UGA recoding activities in vivo and in vitro, spanning over a range of several thousand and hundred fold, respectively. The other pivotal RNA element, the Sec-tRNA ${ }^{[\mathrm{Ser}] \mathrm{Sec}}$, has a particular aminoacylation mechanism. Indeed, the $\mathrm{RRNA}^{[\mathrm{Ser}] \mathrm{Sec}}$ is initially charged with a Ser which is subsequently modified in Sec using selenophosphate and three enzymes (REF). The levels of Sec-tRNA ${ }^{[\mathrm{Ser}] \mathrm{Sec}}$ are highly dependent on the Se bioavailability. Due to this particular biosynthesis pathway, it was recently reported that Cys could substitute Sec during Se deficiency, as thiophosphate could replace selenophosphate during enzymatic reactions. These findings reveal that, at least in mammal selenoprotein mRNAs, UGA can be recognized as stop, Sec or Cys codon, depending on the Se bioavailabitily (ref Hatfield, gladyshev).

Se deficiency causes its redistribution. In every tissue or cell line, the use of $\mathrm{Se}$ is prioritized within the selenoproteome, maintaining essential selenoproteins at the expense of others. This has been well characterized for $\mathrm{Gpx}$ members in rodents (Bermano, Sunde, etc), but remains to be investigated for other proteins of the selenoproteome. The hierarchy of selenoprotein expression is controlled by the bioavailability of Se from food or from culture media. It depends on the tissue and, presumably, on the pathophysiological conditions. In addition to the Se-dependent regulation, selenoprotein expression was found to be differentially regulated by other stimuli, including oxidative stress (Papp, Touat) and by replicative senescence (Legrain) as reviewed elsewhere (Touat et al Selenium book).

The detection of selenoproteins has been long been limited to either the use of gammaradioactive ${ }^{75} \mathrm{Se}$ followed by $1 \mathrm{D}$ gel electrophoresis autoradiography or to immunological methods (mostly westerm immunoblot). However, although successful, immunoblots are limited by the availability of antibodies. Also, they do not provide 
information about the relative abundance of each selenoprotein (Chapter book Michalke). Alternatively, other detection strategies have been elaborated. For example, Se-specific signal produced from selenoproteins can be detected and quantified by inductively coupled plasma mass spectrometry (ICP MS) with the absolute sensitivity of $10^{-15} \mathrm{~g}$ Se. An analytical method allowing the simultaneous detection of several selenoproteins expressed at trace levels by laser ablation of IEF strips coupled with ICP MS detection (LA-ICP MS) was developed (REF Bianga et Bianga, MiMB). The ${ }^{78} \mathrm{Se}$ electropherogram of the IEF strip allows the detection of 10 to 12 peaks from cellular extracts, which in most of the cases were attributed to selenoproteins. This novel strategy based on LA-ICP MS appears complementary to western blots, and represents a possible alternative to gamma radioactivity measurements.

Here, we investigate the selenoproteome hierarchy in four types of cell lines in response to changes in the Se concentration levels in a culture medium, using a set of complementary methodologies. Semi-quantitative and quantitative results were obtained for selenoproteins in terms of mRNAs abundance, protein activity (when available), protein immunodetection, Se-specific detection and SECIS-dependent UGA-Sec recoding efficiency.

\section{EXPERIMENTAL PROCEDURE}

Material and chemicals-The different cells lines used in this study were purchased from Life technologies (HEK293) and ATCC (LNCaP, $\mathrm{HaCaT}$ and HepG2). Luciferase reporter plasmids were generated in (REF Latreche 2009, 2012). Cell culture media, fetal calf serum (FCS) and supplements were purchased from Life Technologies. Transferrin, insulin, 3,5,3'triiodothyronine, hydrocortisone, t-BHP, NADPH, thioredoxin, L-GSH, Glutathione reductase, DTNB, sodium selenite and DTT were purchased from Sigma-Aldrich. Antibodies were purchased from Abcam (Gpx1, \#ab108429; Gpx2, \#ab140130; Gpx4, \#ab125066; SelenoF, \#ab124840; SelenoM, \#ab133681; SelenoO, \#ab172957; SelenoP, \#ab109514), GeneTex (SelenoW, \#GTX48717), Thermo-Scientific (TrxR1, \#LF-MA0015) and Sigma-Aldrich
(SelenoS, \#HPA010025; TrxR1, HPA003323; Actin, \#A1978; Tubulin, \#T9026). Urea, thiourea, CHAPS, Destreak Reagent, IPG Buffer ( $\mathrm{pH}$ 3-10 NL) Immobilin Dry-Strip ( $\mathrm{pH} \mathrm{3-10}$ $\mathrm{NL}, 18 \mathrm{~cm}$ ) and bromophenol blue were purchased from GE Healthcare. NuPAGE 4-12\% bis-Tris polyacrylamide gels, MOPS and MES SDS running buffer were purchased from Life Technologies. Antiprotease inhibitor cocktail was purchased from Thermo Fisher (formerly Proteoblock, Fermentas). Natural and isotopically enriched selenite $\left({ }^{76} \mathrm{Se}\right.$ and $\left.{ }^{77} \mathrm{Se}\right)$ were purchased from SIGMA and Isoflex (Moscow, Russia), respectively.

Cell culture-Cells were grown and maintained in $75 \mathrm{~cm}^{2}$ plates in Dulbecco's Modified Eagle Medium (D-MEM) for HEK293, LNCaP and HaCaT cells and MEM for HepG2 cells. Media were supplemented with $10 \%$ fetal calf serum (FCS), $100 \mu$ g.mL-1 streptomycin, 100 UI.mL-1 penicillin, $1 \mathrm{mM}$ sodium pyruvate, $2 \mathrm{mM}$ L-glutamine. Cells were cultivated at 37 ${ }^{\circ} \mathrm{C}$ in humidified atmosphere containing $5 \%$ of $\mathrm{CO}_{2}$. The different culture media referred to as Ctl, Dpl, Dpl+Se and Sup were generated according to (REF Touat, Legrain, Latreche 2012) and references therein. Selenium concentration was determined by ICP-MS in the FCS lot used throughout the experiments. Selenium concentration in the Ctrl medium was $15 \mathrm{nM}$. In the Dpl medium, we expected $3 \mathrm{nM}$ Se since $2 \%$ FCS was used instead of $10 \%$. To cope with the decrease of growth factors in Dpl medium, $5 \mathrm{mg} . \mathrm{L}^{-1}$ transferrin, $10 \mathrm{mg} . \mathrm{L}^{-1}$ insulin, $100 \mathrm{pM} 3,5,3^{\prime}$-triiodothyronine (T3), and $50 \mathrm{nM}$ hydrocortisone were added, as described and validated for selenoprotein expression studies in (REF Touat, Legrain, Latreche 2009, 2012). An addition of $30 \mathrm{nM}$ sodium selenite to Ctl or Dpl media gave a final Se concentration of 45 and 33 $\mathrm{nM}$ in Sup and Dpl+Se media, respectively. Cellular protein extracts were harvested after $72 \mathrm{~h}$ in $300 \mu \mathrm{L}$ passive lysis buffer containing 25 $\mathrm{mM}$ Tris phosphate $\mathrm{pH} 7.8,2 \mathrm{mM}$ DTT, $2 \mathrm{mM}$ EDTA, $1 \%$ Triton X100 and 10\% glycerol. Then protein concentrations were measured using the DC kit protein assay kit (Biorad) in microplate assays.

Measure of the total Se and enzymatic activities-For the total Se determination, cellular extracts were digested with $\mathrm{HNO}_{3}$ and $\mathrm{H}_{2} \mathrm{O}_{2}$ 
overnight at room temperature as described in Bianga 2014. The Se concentration was measured in a $100-\mu \mathrm{L}$ aliquot by ICP MS (Agilent 7700) in flow injection analysis mode using external calibration. Gpx and TxrR activities were measured in enzymatic coupled assay as described in (REF Touat, Legrain 2014) with $50 \mu \mathrm{g}$ of protein extracts. Gpx enzymatic activities (U/mg) were expressed as nmol of glutathione. $\mathrm{min}^{-1} \cdot \mathrm{mg}^{-1}$. TrxR enzymatic activities (U/mg) were expressed as nmol of NADPH.min ${ }^{1} . \mathrm{mg}^{-1}$. The enzymatic assays were performed in duplicate for three independent experiments.

RNA extraction and real-time PCR-Total RNAs were extracted using Nucleospin RNA II kit (Macherey Nagel). Synthesis of cDNA was carried out using Transcriptor High Fidelity cDNA Synthesis kit (Roche Applied Science) according to the manufacturer's instructions. Real time PCR was performed in triplicate using LightCycler® 480 SYBR Green I Master (Roche Applied Science). The PCR program was $95^{\circ} \mathrm{C}$ for $5 \mathrm{~min}$, and 45 cycles of $95^{\circ} \mathrm{C}$ for $10 \mathrm{sec}$, $60^{\circ} \mathrm{C}$ for $20 \mathrm{sec}, 72^{\circ} \mathrm{C}$ for $20 \mathrm{sec}$. Data were analyzed using LightCycler ${ }^{\circledR} 480$ software and normalized relative to the mRNA levels of Hrpt. The individual abundance of mRNA was arbitrary expressed relative to the total amount of selenoprotein mRNAs set as 100 . The primers used are described in (REF Touat, Legrain 2014).

Protein gels and western immunoblottingEqual protein amounts $(50 \mu \mathrm{g})$ were separated in Bis-Tris NuPAGE Novex Midi Gels and transferred onto nitrocellulose membranes using iBlot ${ }^{\circledR} \quad$ DRy blotting System (Life Technologies). Membranes were probed with primary antibodies (as indicated) and HRPconjugated anti-rabbit or anti-mouse secondary antibodies (Sigma). The chemiluminescence signal was detected using the ECL Select Western Blotting Detection Kit (GE Healthcare) and the PXi 4 CCD camera (Ozyme). Image acquisition and data quantification were performed with the Syngene softwares, GeneSys and Genetools, respectively.

Separation of proteins by isoelectric electrofocusing (IEF)-An aliquot of $500 \mu \mathrm{g}$ of protein extracts was precipitated with chloroform/methanol. Protein pellets were resolubilized in $340 \mu \mathrm{L}$ of rehydration solution containing 8M urea, 2M thiourea, 2\% (w/v) CHAPS, $12 \mu \mathrm{l} . \mathrm{ml}-1$ destreak reagent, $0.5 \%(\mathrm{v} / \mathrm{v})$ IPG Buffer (3-10) and traces of bromophenol blue. This solution was loaded on an $18-\mathrm{cm}$ immobiline dry strip (3-10 NL) by passive rehydration under layer of mineral oil overnight. Electrophoresis was performed on IPGPhor2 (GE Healthcare) using the following programme: $150 \mathrm{~V}(1 \mathrm{~h}), 200 \mathrm{~V}(1 \mathrm{~h})$, gradient $200-1000 \mathrm{~V}$ $(8 \mathrm{~h})$, gradient $1000-8000 \mathrm{~V}(4 \mathrm{~h})$, and $8000 \mathrm{~V}$ (4 h) (Le voltage horaire total ou VhT ne doit pas dépasser 35000). After migration, the proteins in gel strip were fixed in a solution containing 10\% glycerol and 30\% ethanol, and dried under atmospheric conditions.

Laser ablation of IEF strips coupled with ICP MS detection (IEF - LA-ICP MS)-A 213 nm laser (NewWave, UP-213) was coupled with ICP MS (Agilent 7700) as described in Bianga 2011 and Bianga 2014. To fit in the ablation cell, IEF strips were divided in five pieces and placed on glass slide. The scanning was performed at

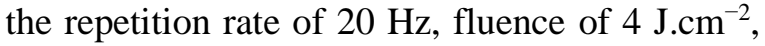
spot size of $250 \mu \mathrm{m}$, and scan speed of $100 \mu$ $\mathrm{m} . \mathrm{s}^{-1}$. Ablated material was carried to ICPMS by $500 \mathrm{~mL} \mathrm{~min}^{-1}$ flow of He mixed just before the torch entrance with $760 \mathrm{~mL} \cdot \mathrm{min}^{-1}$ of Ar gas. ICP MS was used in the collision cell mode using $\mathrm{XX} \mathrm{ml.min}{ }^{-1}$ of $\mathrm{He}$ as the collision/reaction gas. A $1.5 \mathrm{~mm}$ i.d. injector torch and Pt cones were used. The spectrometer was tuned off-line for the maximum Se signal-to-noise ratio. Acquisition time per strip was approximately $40 \mathrm{~min}$.

Data treatment missing

Evaluation of selenocysteine insertion efficiency-To analyze Sec insertion efficiency in HEK293, we used luciferase-based reporter constructs which were validated for UGA/Sec recoding in transfected cells (REF). Briefly, the 26 human SECIS elements were cloned downstream of a luciferase coding sequence, which had been modified to contain an in frame UGA codon at position 258 (Luc UGA/SECIS). Cells were plated in $100 \mathrm{~mm}$-diameter flask the day prior to transfection in Dpl or Dpl+Se media, respectively. Calcium precipitation method was used according to (Latreche et al 2009) to transiently transfect the cells with $6 \mu \mathrm{g}$ of plasmid $(5 \mu \mathrm{g}$ of luciferase and $1 \mu \mathrm{g}$ of $\beta$ galactosidase vectors). The medium was changed 24-h after transfection and cells were 
collected after $48 \mathrm{~h}$ with $300 \mu \mathrm{l}$ of a passive lysis buffer. Then cellular extracts were assayed for luciferase and $\beta$-galactosidase activities by chemiluminescence (Promega Luciferase and Beta-Glo assay systems, respectively), in triplicate using a microplate reader FLUOstar OPTIMA (BMG Labtech). We arbitrarily expressed the Sec insertion efficiency relative to the maximum activity measured for a Luc UGA/SECIS construct in Dpl conditions, i.e. Luc UGA/Dio2, which was set as $100 \%$.

\section{RESULTS}

Se supplementation of the culture medium increases intracellular Se content

In order to examine the regulation of the selenoproteome in relevant cell lines, we have performed a comprehensive study supplementing with Se a culture medium referred to as control (Ctrl) and expected to be deficient in Se $(15 \mathrm{nM})$ to produce conditions referred to as supplemented (Sup) ( $45 \mathrm{nM}$ of total Se). The cell lines used originated from kidney (HEK293), prostate (HepG2), liver (HepG2) and skin (HaCaT) tissues evoked in the context of Se functions in man. The variation of Se levels between Ctrl and Sup conditions were described to affect several selenoproteins in cellular extracts of HEK293 cells, including Gpx1, Gpx4, SelenoS, SelenoK, TxnRD1 and SelenoM (ref touat JBC). The impact of $\mathrm{Se}$ supplementation of the culture medium is first investigated by measuring the total Se uptake by each of the cell lines. Figure 1 shows that a moderate, however significant, increase in the Se uptake occurs in response to a three-fold increase in the Se concentration in the culture medium. The uptake order (from the highest to the lowest) is HEK293 > LNCaP > HepG2 and HaCaT, with respectively a 2.7, 2.0, 1.4 and 1.3 fold-increase in the Se content of cellular extracts in the Sup conditions (compared to Ctrl). Our results indicate that conditions for optimal selenoprotein synthesis require only moderate intracellular accumulation of Se.

\section{Selenoprotein mRNAs are poorly regulated in response to changes in the Se concentration in a culture medium}

In animal models and cultured cell lines, a variation of the selenium content has been shown to selectively alter a limited subset of selenoprotein mRNAs, including Gpx1, SelenoH, SelenoW and Gpx4 mRNAs (REF Chavatte, Sunde, Bermano). However, this response is highly dependent on the animal tissue or the cell line studied. A comprehensive analysis of the steady state levels of the 25 selenoprotein mRNAs by RT-qPCR allows us to identify, if any, Se-sensitive mRNA transcripts in HEK293, LNCaP, HaCaT and HepG2 cell lines. As shown in Figure 2, only a limited and cell-specific response is observed at the mRNA levels. While SelenoW mRNA levels are positively regulated by $\mathrm{Se}$ in three cell lines (LNCaP, HaCaT and HepG2), Gpx1, SelenoP, SelenoV and Gpx3 mRNA levels are modified only in one or two of the cell lines studied. Overall, our data confirm that only a limited regulation of the selenoproteome occurs at the level of mRNA abundance, suggesting, as observed elsewhere (ref), a tighly controlled regulation of selenoprotein expression at the stage of translation.

\section{Response of Gpxs in various cell lines to Se concentration change in a culture medium}

Gpx has been the mostly studied group of selenoproteins yet. Gpxs include five selenoproteins (Gpx1, Gpx2, Gpx3, Gpx4 and Gpx6), with Gpx1 and Gpx4 being ubiquitously expressed in mammals, in cytosol and mitochondria, respectively. So far, only Gpx4 was found essential in animals, since gene inactivation in mice led to embryonic lethality. Gpxs are highly sensitive to the fluctuation of Se concentration and are therefore considered as "stress-related selenoproteins". Here, we have compared the response of Gpx expression to Se supplementation among the different cell lines. Enzymatic activities, mRNA and protein levels are assayed as shown in Figure 3. First, as expected, Gpx activities are strongly stimulated in Sup conditions as compared with Ctrl in all the cell lines tested. Again, from the most to the least responsive, we find a similar ranking of cell lines to that discussed in the context of the of total intracellular Se content, i.e. HEK293 > LNCaP > HaCaT and HepG2 with a 4.3-, 3.8-, 2.2- and 2.0-fold increase in Gpx activity, respectively (Figure 3A). When we analyze mRNA transcripts from the Gpx group, we 
notice that Gpx1 and Gpx4 mRNAs are indeed present at high levels in the four cell lines. The other members, including Gpx2, Gpx3 and Gpx6 are cell-specifically expressed. Remarkably, Gpx2 mRNAs, which are barely detectable in HEK293 or LNCaP, are present in high amount in the HaCaT or HepG2 cell lines (Figure 3B). Concerning the sensitivity to Se supplementation at the mRNA levels, the only variation is observed for Gpx1 and Gpx2 in HepG2 and $\mathrm{HaCaT}$ cell extracts, respectively.

Contrarily to mRNA levels, Gpx protein levels are strongly dependent on the $\mathrm{Se}$ availability, as illustrated in Figure 3C. This is particularly the case for Gpx1, which is stimulated between 4-and 20-fold, depending on the cell lines. As previously reported in HEK293 cells (Latreche 2012, Touat 2014), the upregulation of selenoprotein expression results from an enhancement of translation efficiency, mostly at the stage of UGA recoding. Here, we extend this observation to other cell lines. Interestingly, while Gpx1 is more responsive than Gpx4 to changes in the Se concentration in HEK293 and LNCAP cells, they have a comparable regulation in $\mathrm{HaCaT}$ and $\mathrm{HepG} 2$, notably where Gpx2 is present (Figure 3C). Concerning Gpx2, it appears that a Se-dependent translational control only occurs in HepG2, since in $\mathrm{HaCaT}$, mRNA and protein levels vary similarly. Taken together, our results show that Gpx levels are strongly stimulated by the $\mathrm{Se}$ bioavailability in every cell line tested, essentially via an activation of translation efficiency. Gpx confirm their status of "stressrelated" selenoproteins.

\section{Response of TxnRDs in various cell lines to Se concentration variation in the culture medium}

TxnRDs are also well known selenoproteins, with either a ubiquitous (for TxnRD1 and TxnRD2) or test-specific (for TxnRD3) distribution. They are considered as "house-keeping selenoproteins" and therefore their levels are often poorly affected by Se concentration variation. However, it was recently discovered that under Se deficiency conditions, Cys can substitute $\mathrm{Sec}$ in the catalytic site of TrxRs (Ref Hatfield). Therefore, total levels of
TrxR could stay unaffected on western blots with a strong variation of its specific activities.

A similar comparison as for Gpxs is performed for the different members of TxnRD family among the four cell lines studied. Enzymatic activities, mRNA and protein levels are assayed as illustrated in Figure 4. In Ctrl conditions, it appears that TrxR enzymatic activities are similar in HEK293, HaCaT and HepG2 cells, and approximately five-fold higher in $\mathrm{LNCaP}$ (Figure 4A). Interestingly, in comparison with Gpxs, only a weak but significant increase in TrxR activities in response to $\mathrm{Se}$ supplementation is noticed in HEK293, LNCaP and HepG2, but not in HaCaT. However, as for the Gpx activities, this change is not due to a modification of TxnRD mRNA transcript levels, but only to an increase at protein levels (Figure 4B and C). Therefore, our data indicate that Se supplementation enhances translation efficiency of TxnRD1 and TxnRD2 mRNAs in virtually all the four cell lines studied. It seems that the higher TxnRD activities observed in $\mathrm{LNCaP}$ result from high levels of both TrxR1 and TrxR2 proteins (Figure 4).

Response of other selenoproteins in various cell lines to Se concentration variation in the culture medium

Even though the cellular localization of selenoproteins is often known, enzymatic activities, interacting partners and selenium regulation remain unclear for approximately half of them. Here, we focus on six significant selenoproteins, namely SelenoS, Sephs2, SelenoF (also known as Sel15 and Sep15), SelenoP, SelenoW and SelenoM. Since little is known about their ranking in the hierarchy of selenoproteins, we investigate their levels of mRNA transcripts and proteins in Ctrl and Sup conditions (Figure 5). At the transcript levels, only SelenoW has an even and positive response to Se supplementation in all the cell lines tested. Our observation is in agreement with Sunde et al. which finds SelenoW mRNA levels as one marker of Se status in rodents (REF). However, little is known about its function, SelenoW is mostly expressed in brain and muscle and has a redoxin-fold motif (C-X-X-U) presumably in its catalytic site. Concerning SelenoW, it appears 
that protein levels reflect mRNA levels, suggesting a poor, if any, translational control of its expression in the cell lines tested (Figure 5). Also, other selenoprotein mRNAs do not appear to be altered by the Se supplementation, except for SelenoP mRNA, which is approximately reduced by half in HepG2 cells cultured in Sup conditions.

On the protein level, only several selenoproteins are regulated by the variation of $\mathrm{Se}$ concentration in the medium, namely SelenoS, SelenoF, SelenoW and SelenoM, and can therefore rank as "stress-related selenoproteins". On the other side, Sephs2 and SelenoP are poorly regulated by selenium concentration variation (Figure 5) and could be associated with housekeeping selenoproteins. Our data indicate that in the cell lines analyzed here, a selective translational control of selenoprotein occurs. Remarkably, as summarized in Table 1, the hierarchy of the selenoproteome seems cell-specific.

\section{Alteration of the selenoproteome profile in response to a Se supplementation analyzed by IEF - LA-ICP-MS}

Data about relative abundance of individual selenoproteins is essential to precisely characterize the redistribution of Se within the selenoproteome when the Se concentration varies in the culture media. This important information is lacking from the western blots but can be obtained from by measuring the signal of Se present in selenocysteine residues by ICPMS.

We have recently developped an innovative method to analyse the relative amount of abundant selenoproteins in cellular extracts (REF Bianga). First, proteins are separated according to their isoelectric point (pI) on IEF gel strips designed with an immobilized $\mathrm{pH}$ gradient. We use a broad and non linear gradient, from $\mathrm{pH} 3$ to 10 , to detect the maximum number of selenoproteins. Then, after electrophoretic migration of the proteins to their respective pI, proteins are fixed in the gel and the IEF strips are scanned for the Se signal by LA-ICP-MS, as illustrated in Figure 6A (and REFs). Only Se containing proteins are detected. The electropherograms obtained are representative of the respective abundance of the $\mathrm{Se}$ signal between the different selenoproteins, especially since only one selenium atom is present per selenoprotein (except for SelenoP, which has ten selenocysteine residues in human). The identity of the most intense Se-peaks (SelenoF, Gpx1, TxrR1, TxnRD2 and Gpx4) was previously determined by proteomic analysis (REF Bianga). Note that this method allows us to separate several isoforms of TxnRD1, TxnRD2 and Gpx4 (REF Bianga).

Here, we have optimized the methodology by using a selenium enriched isotope $\left({ }^{76} \mathrm{Se}\right)$ to increase the signal detected by ICP-MS. Indeed, Se has 6 stable isotopes: ${ }^{74} \mathrm{Se}(0.89 \%),{ }^{76} \mathrm{Se}$ $(9.37 \%),{ }^{77} \mathrm{Se}(7.63 \%),{ }^{78} \mathrm{Se}(23.77 \%),{ }^{80} \mathrm{Se}$ $(49.61 \%)$ and ${ }^{82} \mathrm{Se}(8.73 \%)$. Often with ICP-MS, the measurement of ${ }^{78} \mathrm{Se}$ signal is chosen for selenium detection since the signal of most abudant isotope, i.e. ${ }^{80} \mathrm{Se}$, is highly interfered by ${ }^{40} \mathrm{Ar}$ dimers from the plasma gas. However, ${ }^{78} \mathrm{Se}$ represents only one fourth of total selenium present in each selenoprotein when natural selenite is used. Interestingly, each of Seisotopes can be enriched to $>99 \%$ in selenite and used in cultured media to label and trace selenoprotein expression by ICP-MS (REF mimb). We have applied this labeling strategy to the four cell lines discussed here.

HEK293, LNCaP, HaCaT and HepG2 cell lines are grown in depleted medium $(\mathrm{Dpl})$ with 10 or $30 \mathrm{nM}$ of ${ }^{76}$ Se-enriched selenite, corresponding to Ctrl and Sup conditions, respectively. Therefore, most of Se signal should be concentrated on ${ }^{76} \mathrm{Se}$ signal within selenoproteins as shown in (ref mimb). Then, protein cell extracts are migrated on IEF strip which are further scanned by LA-ICP-MS. As shown in Figure 6, the methodology allows a reliable alignement of Se peaks. A maximum of 12 Se peaks can be distinguished in the electropherograms. Among them, 9 could be attributed to known selenoproteins. The other three peaks, namely peak 2, 3 and 4 could not be unambiguously identified due to the lack of analytical sensitivity (see REF Bianga), but given their theoritical $\mathrm{pI}$, they are likely to correspond to SelenoN (pI 5.38), SelenoM (pI 5.39), Sephs2 (pI 5.63) or SelenoO (pI 5.69). The peak areas are integrated and reported in Table 2 for all the conditions tested. 
As it has been shown in Figure 6 and Table 2 , several selenoproteins are detected in all four cell lines, which include peak 1 (SelenoF), peak 3 (Sephs2), peak 4 (SelenoO or SelenoM), peak 5 (Gpx1) and peak 7 (TrxR1). Gpx1 appears as the most abundant selenoprotein in HEK293 and $\mathrm{HaCaT}$, and among the most abundant proteins in LNCAP and HepG2, confirming that Gpx1 is an ubiquitous selenoprotein in human cells. As expected, the relative abundance of selenoproteins is specific to each cell line : (i) in HEK293, we have Gpx1>SelenoF>peak2 (SelenoN or SelenoM)> TxnRD1; (ii) in LNCaP, we have TxnRD1>Gpx4>Gpx1> TxnRD2>SelenoF; (iii) in HaCaT, we have Gpx1>Gpx4>SelenoF> TxnRD1; (iv) in HepG2, we have Gpx4>Gpx1> SelenoF> TxnRD1. This data confirms previous observation that TxnRDs are highly expressed in LNCaP. Additionally, the quantitative integration of the ${ }^{76} \mathrm{Se}$ electropherograms shown in Table 2, provides us with information about the hierarchy of selenoprotein expression in different cell lines in response to Se supplementation.

Efficiency of the selenocysteine insertion into selenoprotein is modulated by Se levels and depends on the nature of the SECIS

As noted earlier, several selenoproteins respond differentially to a Se supplementation, presumably via a translational control of the UGA/Sec recoding event. Indeed, selenocysteine insertion is not only a crucial stage of selenoprotein mRNA translation, but also an important regulatory checkpoint for selenoprotein expression. Remarkably, several factors and stimuli modulate selenocysteine incorporation by regulating UGA recoding efficiency. Among them Se levels and the nature of the SECIS element are important regulators. We have developed a complete set of luciferase based reporter constructs that allows the investigation of SECIS influence on UGA recoding. All 26 SECIS found in human genome were individually cloned downstream of luciferase constructs. In thist study, we transfect the LucUGA/SECIS plasmids in HEK293 cells grown either in Dpl or Dpl supplemented with $30 \mathrm{nM}$ of $\mathrm{Se}$ (Figure 7).

As noted in REF, SECIS element influences considerably the recoding efficiency. Here we find that this holds true when cells are grown in $\mathrm{Dpl}$ and Dpl+Se conditions. Strikingly, the range of UGA recoding efficiencies between the weakest and strongest constructs is much more important in Se supplemented conditions (Dpl+Se) than in Dpl (Figure 7, compare A and B). Interestingly, when focusing on individual constructs, it is worth-noting that the extent of stimulation due to Se supplementation is strongly dependent on the nature of the SECIS element. Our data strongly suggest that the presence of a weak SECIS element results in a poor stimulation by $\mathrm{Se}$ supplementation in comparison with constructs with a strong element.

Taken together, our data indicate that the SECIS element dictates the selenocysteine insertion efficiency and, especially, its response to the variation of the Se concentration in a culture medium.

\section{DISCUSSION}

In this work, we report the hierarchical regulation of the selenoproteome in human cancerous (LNCaP and HepG2) and immortalized (HEK293 and HaCaT) cell lines, which is relevant to the selenium biological properties. We have analyzed this regulation in response to selenium variation in a comprehensive approach at the levels of mRNAs, proteins, enzymatic activities and presence of covalently bound Se. It appears that the selenoprotein hierarchy is cell-line specific with some conserved features.

Selenoproteome analysis by IEF - LAICP-MS provides complementary information to western blot assays

In contrast to other proteins, selenoproteins have the intrinsic property to contain a covalent $\mathrm{Se}$ atom in their primary structure which is detectable by ICP-MS. Using this feature, we have developed a novel methodology to analyse selenoproteome expression in cellular extracts without the need for the ${ }^{75} \mathrm{Se}$ radioactive isotope. Although this technology is far from routine use it can be applied to specific analyses in dedicated research laboratories. In this study, we improve our strategy by using a ${ }^{76} \mathrm{Se}$ enriched isotope to label and trace newly synthesized selenoproteins in growing cells by IEF - LA-ICP-MS. A 
maximum of 12 selenoproteins could be analysed in cellular extracts, which is at least as many as with the use of radioactive isotopes followed by autoradiography of SDS-PAGE gels.

A common feature between radioactive and stable isotope labeling is the detection of Se atom which is fundamental for the enzymatic activity of selenoprotein. This information is crucial since it has been evidenced that Cys could substitute Sec in selenoproteins in cultured cells and in mice mostly during Se deprivation. Further investigations are needed to characterize Cys replacement of Sec in Dpl conditions. Concomitant analyses by western blots and LAICP-MS would definitely be useful to address this issue. However, the current limitation of LA-ICP-MS strategy is the detection limits for Se which are expected to be improved by the next generations of instrumentation.

An important feature of ICP-MS is the possibility of simultaneous detection of several isotopes (REF Lobinski Selenium book). For Se, six stable isotopes can be enriched as selenite, used to label selenoprotein, and detected simultaneously by ICP-MS. It implies that different cell lines or different treatments of the same cell line could be labeled with specific Seisotopes, mixed and analysed for in one IEF for quantitative and multiplexed analyses.

\section{Selenoprotein hierarchy is strongly dependent on the type of cell line}

Table 1 and Table 2 report the hierarchy of selenoprotein stimulation by Se supplementation in the different cell lines tested here, as revealed by western immuno blots or by IEF - LA-ICPMS, respectively. Western immunoblotting demonstrates that Gpx1 and Gpx4 are the most sensitive proteins to Se concentration variation in HEK293, LNCaP and HaCaT, and rank high in the hierarchy of selenoproteome. For HepG2, the hierarchy is drastically different with SelenoF, SelenoM and SelenoS higher in the ranking than Gpx1 and Gpx4. Interestingly, these three proteins are located in the ER where they are involved in protein-folding and ERassociated degradation (ERAD). Whether the function of the ER is affected by the Se levels in HepG2 remains to be investigated.
IEF - LA-ICP-MS explores selenoprotein expression from a different angle than detection using antibodies since it focuses only on the presence of Se. Interestingly, the results from the two detection methods are slightly different. IEF - ICP-MS indicates that selenoproteins are slightly less sensitive to selenium concentration variation than does the detection using antibodies (cf. Tables 1 and 2). The regulation of Gpx1 by Se concentration variation illustrates this finding. Although Gpx1 is always positively responding to a Se-supplementation, the fold stimulation is larger when observed in western blots. What clearly appears from the ${ }^{76} \mathrm{Se}-$ electrophoregrams is the selective response of certain selenoproteins at the expense of the others. Altogether, our results confirm that the selenoprotein hierarchy is cell-line specific, with a wide range of stimulation. This novel strategy for selenoprotein analysis paves the way for further studies on Se redistribution in response to various exogenous stimuli or pathophysiological conditions.

\section{Selenoprotein hierarchy is mostly regulated by translation efficiency of UGA recoding \\ The mechanism of selenoprotein} biosynthesis is unique since $\mathrm{Sec}$ is encoded by a UGA codon, which is generally recognized as a termination signal for protein synthesis. This stage of UGA-Sec recoding is rate-limiting and is used as a regulatory checkpoint by the cell (REF Bulteau-Chavatte). The efficiency of Sec insertion is modulated by different factors, one of which is the nature of the SECIS element. Here we find that in a luciferase-reporter construct, the presence of a weak SECIS element (such as, e.g. SelenoO, Gpx6, SelenoS or Gpx3) results in a poor regulation of UGA recoding efficiency by selenium concentration variation. On the other hand, the other SECIS elements make the UGA recoding event strongly dependent of selenium levels. For example, the efficiencies of the SECIS elements from Gpx1, Gpx2 and Gpx4, considered as "stress-related selenoproteins", are much more sensitive to $\mathrm{Se}$ concentration variation than the ones of TrxR1, TrxR2 and Sephs2, considered as "housekeeping selenoproteins". 
The classification between strong and weak SECIS elements has been previously reported (latreche), and depends on the SECIS core region, which is the binding site of several factor. However, the intimate mechanism for this distinction is elusive and may rely on additional cellular factors or intrinsic RNA-folding features. 


\section{REFERENCES}

\section{FOOTNOTES}

\section{FIGURE LEGENDS}

Figure 1. Intracellular Se levels in Hek293, LNCaP, HaCaT and HepG2 cells in Ctrl and Sup conditions.

Figure 2. Evolution of selenoprotein mRNA levels as a function of Se concentration in Hek293, LNCaP, HaCaT and HepG2 cell lines.

For clarity, only mRNA level changes higher than two-fold are represented in light grey.

Figure 3. Comprehensive study of Se-dependent regulation of Gpxs in Hek293, LNCaP, HaCaT and HepG2 cells.

Figure 4. Comprehensive study of Se-dependent regulation of TrxRs in Hek293, LNCaP, HaCaT and HepG2 cells.

Figure 5. Se-dependent regulation of other important selenoproteins.

Figure 6. Study of Se-dependent regulation of the selenoproteome by IEF - LA-ICP MS.

Figure 7. The response of UGA/Sec recoding efficiency as a function of selenium levels depends on the nature of the SECIS element. 


\section{TABLES}

Table 1. Hierarchy of Selenoenoproteins in HEK293, LNCaP, HaCaT and HepG2 as reavealed by western immuno blots. n.d., not detected.

\begin{tabular}{|c|c|c|c|c|c|c|c|c|c|c|c|c|c|c|c|}
\hline \multicolumn{4}{|c|}{ HEK293 } & \multicolumn{4}{|c|}{ LNCaP } & \multicolumn{4}{|c|}{ HaCaT } & \multicolumn{4}{|c|}{ HepG2 } \\
\hline & $\mathrm{Ctl}$ & Sup & Sup/Ctl & & Ctl & Sup & Sup/Ctl & & Ctl & Sup & Sup/Ctl & & $\mathrm{Ctl}$ & Sup & Sup/Ctl \\
\hline Gpx1 & 5.2 & 100.0 & 19.1 & Gpx1 & 4.0 & 60.3 & 14.9 & Gpx4 & 1.7 & 16.3 & 9.8 & SelenoF & 7.2 & 100.0 & 13.9 \\
\hline Gpx4 & 5.1 & 50.7 & 9.9 & Gpx4 & 4.8 & 28.3 & 5.9 & Gpx1 & 3.0 & 8.5 & 2.8 & SelenoS & 14.6 & 87.9 & 6.0 \\
\hline TxnRD2 & 14.5 & 31.1 & 2.1 & SelenoF & n.d. & 3.8 & $>3$ & Selenos & 39.9 & 86.7 & 2.2 & SelenoM & 3.1 & 14.8 & 4.8 \\
\hline SelenoS & 51.5 & 100.0 & 1.9 & SelenoW & 39.9 & 100.0 & 2.5 & SelenoF & 2.5 & 3.6 & 1.5 & SelenoW & 17.6 & 81.2 & 4.6 \\
\hline SelenoW & 5.0 & 8.1 & 1.6 & SelenoM & 40.5 & 100.0 & 2.5 & TxnRD1 & 2.2 & 2.8 & 1.3 & Gpx1 & 1.0 & 4.3 & 4.4 \\
\hline SelenoF & 7.9 & 12.7 & 1.6 & TxnRD1 & 47.8 & 100.0 & 2.1 & Gpx2 & 77.9 & 99.1 & 1.3 & TxnRD1 & 6.3 & 26.3 & 4.2 \\
\hline TxnRD1 & 6.3 & 9.4 & 1.5 & TxnRD2 & 56.7 & 100.0 & 1.8 & TxnRD2 & 17.5 & 21.2 & 1.2 & Gpx4 & 28.3 & 100.0 & 3.5 \\
\hline SelenoM & 1.3 & 1.8 & 1.4 & Selenos & 30.0 & 49.9 & 1.7 & SelenoW & 23.3 & 23.5 & 1.0 & Gpx2 & 45.4 & 100.0 & 2.2 \\
\hline Sephs2 & 40.9 & 36.2 & 0.9 & Sephs2 & 41.4 & 65.9 & 1.6 & Sephs2 & 100.0 & 80.1 & 0.8 & SelenoP & 22.5 & 23.7 & 1.1 \\
\hline SelenoP & 59.6 & 52.1 & 0.9 & SelenoP & 100.0 & 84.0 & 0.8 & SelenoM & 1.8 & 1.0 & 0.5 & TxnRD2 & 34.5 & 18.3 & 0.5 \\
\hline Gpx2 & n.d. & n.d. & & Gpx2 & n.d. & 2.1 & & SelenoP & 43.7 & 22.0 & 0.5 & Sephs2 & 25.5 & 7.5 & 0.3 \\
\hline
\end{tabular}

Table 2. Hierarchy of Selenoproteins in HEK293, LNCaP, HaCaT and HepG2 as reavealed by LA-ICP-MS of IEF strips

\section{FIGURES}

\begin{tabular}{|c|c|c|c|c|c|c|c|c|c|c|c|c|c|c|c|c|c|c|c|}
\hline \multicolumn{5}{|c|}{ HEK293 } & \multicolumn{5}{|c|}{ LNCaP } & \multicolumn{5}{|c|}{ HaCaT } & \multicolumn{5}{|c|}{ HepG2 } \\
\hline $\mathrm{n}^{\circ}$ & SeProt & $\mathrm{Ctl}$ & Sup & Sup/Ctl & $\overline{n^{\circ}}$ & SeProt & Ctl & Sup & Sup/Ctl & $\overline{n^{\circ}}$ & SeProt & Ctl & Sup & Sup/Ctl & $\mathrm{n}^{\circ}$ & SeProt & Ctl & Sup & Sup/Ctl \\
\hline 5 & Gpx1 & 23.1 & 50.3 & 2.2 & 11 & Gpx4-1 & 2.6 & 9.7 & 3.6 & 5 & Gpx1 & 25.3 & 54.9 & 2.2 & 12 & Gpx4-2 & 3.6 & 22.0 & 6.1 \\
\hline 3 & Sephs2* & 2.9 & 5.2 & 1.8 & 9 & TxnRD2-1 & 2.0 & 6.3 & 3.2 & 4 & SelenoO* & 3.4 & 5.5 & 1.6 & 1 & SelenoF & 6.3 & 14.3 & 2.3 \\
\hline 7 & TxnRD1-2 & 5.6 & 9.9 & 1.8 & 3 & Sephs2* & 2.4 & 7.0 & 2.9 & 12 & Gpx4-2 & 9.2 & 13.3 & 1.4 & 11 & Gpx4-1 & 9.9 & 22.4 & 2.3 \\
\hline 1 & SelenoF & 11.4 & 17.6 & 1.5 & 4 & SelenoO* & 2.4 & 7.2 & 2.9 & 7 & TxnRD1-2 & 6.8 & 9.7 & 1.4 & 8 & TxnRD1-3 & 1.0 & 2.0 & 1.9 \\
\hline 4 & SelenoO* & 4.5 & 6.9 & 1.5 & 8 & TxnRD1-3 & 2.1 & 6.0 & 2.9 & 3 & Sephs2* & 3.8 & 4.7 & 1.2 & 5 & Gpx1 & 12.0 & 20.4 & 1.7 \\
\hline 2 & SelenoN ${ }^{\star}$ or & 8.4 & 10.0 & 1.2 & 1 & SelenoF & 5.2 & 14.1 & 2.7 & 1 & SelenoF & 13.2 & 11.9 & 0.9 & 4 & SelenoO* & 3.8 & 6.2 & 1.6 \\
\hline 6 & TxnRD1-1 & - & - & & 7 & TxnRD1-2 & 4.7 & 12.1 & 2.5 & 2 & SelenoN ${ }^{\star}$ or & - & - & & 2 & SelenoN ${ }^{\star}$ or & 1.5 & 2.3 & 1.6 \\
\hline 8 & TxnRD1-3 & - & - & & 12 & Gpx4-2 & 4.1 & 10.3 & 2.5 & 6 & TxnRD1-1 & - & - & & 3 & Sephs2* & 1.9 & 2.7 & 1.4 \\
\hline 9 & TxnRD2-1 & - & . & & 6 & TxnRD1-1 & 2.9 & 6.1 & 2.1 & 8 & TxnRD1-3 & - & - & & 7 & TxnRD1-2 & 8.4 & 7.8 & 0. \\
\hline 10 & TxnRD2-2 & - & - & & 10 & TxnRD2-2 & 2.6 & 5.0 & 1. & 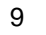 & & - & - & & 6 & D1-1 & - & - & \\
\hline 11 & Gpx4-1 & - & - & & 5 & Gpx1 & 8.6 & 14.4 & 1.7 & 10 & TxnRD2-2 & - & - & & 9 & TxnRD2-1 & - & - & \\
\hline 12 & Gpx4-2 & - & - & & 2 & SelenoN or & 1.4 & 1.9 & 1.3 & 11 & Gpx4-1 & - & 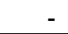 & & 10 & TxnRD2-2 & - & - & \\
\hline & Total & 56 & 100 & 1.8 & & & 41 & 100 & 2.4 & & & 62 & 100 & 1.6 & & & 48 & 100 & \\
\hline
\end{tabular}




\author{
Abbreviations : \\ Ctrl: control \\ DIO: iodothyronine deiodinase \\ DTNB: \\ Dpl: Depleted \\ EFsec: selenocysteine-specific elongation factor \\ ERAD: endoplasmic-reticulum-associated protein degradation \\ Gpx: glutathione peroxidase \\ GSH: glutathione \\ $\mathrm{H}_{2} \mathrm{O}_{2}$ : hydrogen peroxide \\ NADPH: \\ NMD: nonsense mediated decay \\ OPM: oxidative post-translational modifications \\ SBP2: SECIS-binding protein 2 \\ SECIS: selenocysteine insertion sequence \\ shRNA: small hairpin RNA \\ Sup: supplemented \\ ROS: Reactive oxygen species \\ t-BHP: Ter-butyl hydroperoxide \\ TR: thioredoxin reductase \\ UTR: untranslated region
}

